\title{
A qualitative descriptive analysis of nurses' perceptions of hospice care for deceased children following organ donation in hospice cool rooms
}

Short title: Organ donation and children's hospice cool rooms

Michael J Tatterton, Consultant Nurse/Head of Nursing ${ }^{1}$

Rachel Summers, Specialist Nurse Organ Donation ${ }^{2}$

Caroline Y Brennan, Practice Educator ${ }^{1}$

${ }^{1}$ Martin House Children's Hospice, Wetherby, UK

${ }^{2}$ Yorkshire Organ Donation Services Team, NHS Blood and Transplant, Leeds, UK

Corresponding author: Dr Michael Tatterton, MTatterton@martinhouse.org.uk, 01937845045 
Organ donation and children's hospice cool rooms.

\section{Abstract:}

Aim: To explore the perceptions and experience of nursing staff who are involved in supporting families of children and young people who have been cared for in children's hospice cool rooms after death, following organ donation. Background: Following organ donation, bodies of children are generally cared for in hospital mortuaries or by funeral directors, and their families offered little routine bereavement support. A partnership between an organ donation nursing team and regional children's hospice trialled an initiative where families were offered bereavement support from the hospice, and their child's body cared for in a 'cool room' after death. Hospice services are usually restricted to children with life limiting conditions, and their families. Design: A qualitative exploratory study consisting of a focus group interview with registered nurses from the children's hospice and organ donation teams. Review methods: A purposeful sample of nurses was recruited. Data were collected in a digitally recorded focus group interview in March 2018. The interview was transcribed and analysed using a qualitative content approach. Results: Six nurses participated in the focus group. Analysis revealed five themes that characterised the perceptions of nurses: (i) barriers to care, (ii) bereavement care for families, (iii) impact on families and staff, (iv) influencers and enablers of change, and (v) sustainability of new practices. Conclusion: Nurses perceived the longterm, responsive and family centred approach to bereavement support as a strength of the hospice model, reducing the experience of moral distress in organ donation nurses.

\section{Key words:}

bereavement; children; family nursing; focus group; hospice; organ donation 
Organ donation and children's hospice cool rooms.

\section{Introduction}

In the UK, there are in excess of 7,000 people waiting for an organ transplant; 1,000 of these people die each year due to a lack of donated organs (Sque et al. 2018). In the UK between April 2017 and March 2018 there were 1,575 organ donors, 57 of which were under the age of 18 years (NHS Blood and Transplant, 2018); five of these children were from Yorkshire. Little focus has been on the emotional and bereavement support need of families of children during the donation process, particularly post-donation (Berntzen \& Bjørk 2014). There are no published papers that explore the perspectives of nurses who care for the bereaved families of children after organ donation.

There are 53 children's hospices offering a range of services, designed to support neonates, infants children and young people (children) with life limiting conditions (LLC) (Widdas, McNamara \& Edwards 2013), and their families in the UK. Many have the facility to allow the bodies of children to remain in the hospice after death, in 'cool rooms' (Forrester 2008), providing families with an alternative to traditional mortuary facilities.

Specialist Nurses in Organ Donation (SNODs) predominantly have a nursing background in adult critical care. Procedures for the care and support of donors are standardised, regardless of whether they were supporting the family of a paediatric or adult donor. The introduction of a SNOD with a background in children's critical care and palliative nursing to the Yorkshire Organ Donation Services Team led to the realisation that families of children needed time, support and extended bereavement support, provided in a family-centred way. It was known that this level of bereavement care was offered routinely to families within the hospice setting, and that the hospice had recently extended its bereavement support offer to families of children who had died but were not known to the hospice before death. Following a discussion with Martin House Children's Hospice, it was agreed that children who were unknown to the hospice could be referred post- 
Organ donation and children's hospice cool rooms.

donation. This is a move away from the traditional limitations of hospice care, where care is provided to children with LLCs and their families.

Martin House provides palliative care to children with LLCs, and their families across North, East and West Yorkshire in the North of England. Since opening 31 years ago, there have been a small number of children who have donated organs and tissue at the hospice; these children all had LLCs, were known to the hospice, and were receiving care prior to their death. Over the last four years, the hospice has actively sought to engage with stakeholders, aiming to increase reach and awareness of services offered, and to ensure that services provided are relevant, meeting the contemporary needs of children and families, and the services who offer care to them. This has resulted in the broadening of the acceptance criteria which has led to an increase in families whose children died in circumstances not previously seen by the hospice. These include stillborn infants and death in the perinatal period following increases in the number of antenatal referrals, and children referred following catastrophic events such as, life-threatening head injuries, cerebral bleeds, near-drownings, post-meningococcal sepsis and attempted suicides, who have used the hospice for compassionate extubation (Cottrell et al. 2011). The families of these children are able to access the bereavement services of the hospice, which includes specific support groups for parents, siblings (with a specialist group for siblings of babies that died in the perinatal period) and grandparents. In addition to the groups, families are offered an individual bereavement counsellor, who provides support for up to two years after death. Until this initiative, Martin House did not accept referrals for children who had already died to use the hospice cool room.

\section{Aim}

The aim of the study is to explore the perceptions and experience of nursing staff who are involved in caring for the families of children who have been cared for in cool rooms after donating their organs. 
Organ donation and children's hospice cool rooms.

\section{Method}

A semi-structured focus group was conducted with nurses from the Yorkshire Organ Donation Services Team, and Martin House Children's Hospice in March 2018, attended by a combination of SNODs and hospice nurses.

Design

A qualitative descriptive design using a focus group interview was used for this study. Focus groups have been shown to stimulate the sharing of attitudes and opinions on sensitive, under-investigated topics (Shaha, Wenzel \& Hill 2011) and therefore offered an appropriate means of exploring the perceptions of staff involved in caring for the bodies of children, and their families following organ donation; a topic which has not previously been subject to empirical research. SNODs and hospice nurses were brought together in a single focus group to share the experiences of both sets of nurses involved in two different aspects of the child and family's experience. The composition of the focus group matches that of the multiagency awareness raising sessions held jointly for both groups of staff.

\section{Participants}

We anticipated a focus group of four to eight participants. Purposeful sampling was used, based on registered nurses who either:

- Whilst working as a SNOD, referred a child and family to the hospice following organ donation

- Whilst working at the hospice, cared for the body of a child after organ donation, and their family. 
Organ donation and children's hospice cool rooms.

\section{Data collection}

A semi-structured focus group schedule was developed from the topics highlighted by staff during shared education sessions between SNOD and hospice nurses, and professional open events held at the hospice for SNODs. A focus group schedule is shown in figure 1. The focus group was conducted by three researchers, one as convenor (MT), with the others taking notes and interjecting with additional probes as required (CB and RS). Participants, comprising both hospice and SNOD nurses, and researchers sat around a table to facilitate discussion. Participants were encouraged to engage in an open discussion, rather than to merely answer questions.

Thinking about all stages of care (before donation, after donation but before arrival at the hospice, once at the hospice and bereavement follow-up), consider the changes to practice in relation to the following:

- What are the benefits to families?

- What are the challenges?

- What are the benefits to you as a professional?

- What changes have you needed to make to the way you work?

- What have the main learning points been?

- What still needs to be done?

Figure 1: focus group schedule

\section{Ethical considerations}

Health Research Authority (HRA) approval was sought (IRAS Project ID: 235801), however it was concluded that HRA approval was not required. Local approval granted by the hospice board. Participants were informed in writing and verbally that participation in the focus group was voluntary and confidential. Each participant gave informed consent before participation and was reminded to treat the discussion from the focus group confidentially. The shared educating sessions between SNOD and hospice nurses suggested that sensitivity was required during the focus group, aware that they may be discussing experiences that they found challenging and upsetting. This was 
Organ donation and children's hospice cool rooms.

expressed by the convenor at the start of the group, highlighting support available to staff from both organisations.

Data analysis

The focus group was audio recorded, transcribed verbatim and data were analysed using a qualitative content approach to identify emergent themes.

Rigour

All three researchers analysed the focus group transcript and fieldnotes independently before discussing constructs and agreeing the thematic framework. Following data mapping, the authors ensured all relevant data featured in the framework, and that the map represented the data derived from the transcript.

\section{Findings}

The focus group was attended by six participants, each with more than 15 years clinical experience, and a minimum of four years in their current role. All attendees were experienced in supporting families whose children had donated organs and who had been referred to the hospice. Five core themes emerged from the thematic analysis: (i) barriers, (ii) bereavement, (iii) impact, (iv) influencers and enablers, and (v) sustainability. These are shown in figure 2. Quotes from participants are presented to illustrate findings, with words such as 'erm' and 'err' removed. 


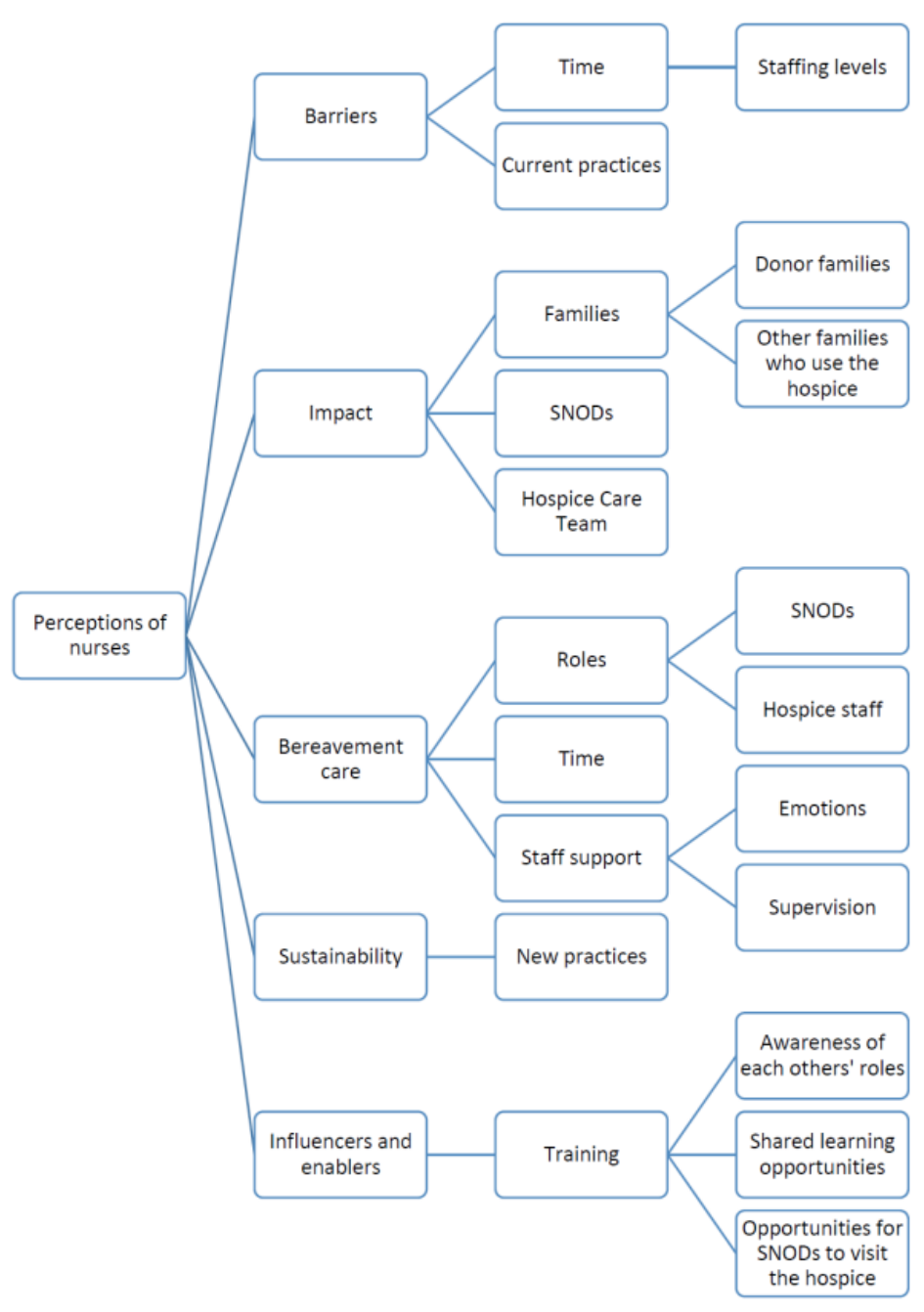

Figure 2: thematic representation of the focus group

\section{Barriers}

Barriers that prevented SNODs from being able to provide the bereavement support to parents following organ donation were highlighted by all SNODs. These barriers were divided into two subordinate themes of time, and current practice restrictions. Although much of the focus of SNOD involvement pre-donation surrounds the emotional support of families, that ends suddenly postdonation. Following the retrieval operation, SNODs will usually support parents and paediatric intensive care unit (PICU) staff to perform last offices, before the child's body is transferred to the hospital mortuary, at which point face-to-face contact with the family usually ends. 
Organ donation and children's hospice cool rooms.

'We don't have regular contact with all of our families.' (SNOD1)

SNODs recognised the complexities of families, discussing separated parents, step-relations, siblings and grandparents. Although it was accepted that ongoing bereavement support was not the role of the SNOD team, the distress at not being able to provide or signpost to bereavement support was highlighted:

'The step siblings were really struggling, so it was a case of advising him to contact his GP I'd phoned [a bereavement charity], they couldn't help because it was less than six month [since death], and I really struggled. Awful. I spent the full afternoon trying to work out what I could do and there was really nothing.' (SNOD1)

Routinely, families are offered a single face-to-face meeting with a PICU consultant 12 weeks after death. Face-to-face contact with SNODs after death is not usual, although it is acknowledged that it happens, described as being 'unofficially expected' (SNOD2).

'Family visits are an unusual thing for us. It's not a regular thing that we do.' (SNOD2)

SNODs provide telephone and letter contact to donor families, usually focused on recipient follow up. Increased donor activity, and changes to the way the team works means that SNODs get less time to focus on follow-up with donor families. Letters to families, once written individually by SNODs, are now based on templates, which appeared to add to the frustrations of SNODs. These changes prompted the SNOD team to contact the hospice to seek more comprehensive bereavement support for families:

'The reason that myself and [the PICU Family Care Sister] have pushed for [the hospice] so much is that because without [the hospice] these families get hardly anything - it's not enough, when they have given so much and are going through such pain. We know you'll support them, when we can't' (SNOD1) 
Organ donation and children's hospice cool rooms.

\section{Bereavement}

The bereavement support offered by both services was discussed, including the differences in roles, focus, time and staff support. Examples of joint working that had already been undertaken were discussed, which highlighted the strengths of each team, and the differing foci taken by the nurses:

'[Mum] is struggling with things like the [organ] recipients haven't written back - things that we can help her with and that maybe [the hospice] can't, but there are things there's not a chance we can help with that [the hospice] can - like ongoing support and helping the whole family grieve.' (SNOD2)

The challenge of supporting families of children who were not known to the hospice, and who died from a sudden event, including brain injuries, cerebral haemorrhage or suicide attempt presented a number of challenges to the hospice care team. The 'different' grief that families experienced, and the subsequent care that they required initially appeared outside of the scope of hospice care. The fact that it was 'unusual for us not to know a family' (Hospice Nurse (HN)1) was highlighted.

'For [the hospice] I suppose, you build up a rapport with families over a period of time if they are coming in for respite and then going home, so by the time the children do die, you've got that [therapeutic relationship], where as with us, the first time you meet that family is because they've lost their child, and usually in traumatic circumstances.' (SNOD2)

Impact

The impact on families, SNODs and hospice staff was discussed from the perspectives of those in the focus group, often alongside specific examples of children and families. The positive impact on families was discussed repeatedly by SNODs, including the responsiveness, personalised and family- 
Organ donation and children's hospice cool rooms.

focused nature of the bereavement care offered by the hospice. This consequently had a positive impact on the SNODs, who felt they were enabling families to receive more appropriate care:

'we would love to give the time; that's why I think it's so - we're so positive as a team about working with [the hospice] because it just means that we know that if we're referring, and if parents want it, that [the hospice] - it's a kind of a weight lifted off our shoulders and you just think 'thank God for that' because they are going to get the care they need.' (SNOD2)

In addition to the emotional and bereavement support offered to families, the care of the child's body was also perceived to benefit families:

'A lot of the families we care for, particularly the paediatric families hate - detest - the thought of their family member going to a mortuary - 'my child's going to be on their own in a mortuary' you know, and for them to know that they are coming somewhere like [the hospice] its just - so good for them.' (SNOD3)

Initially, the impact on hospice staff was not considered to be positive; families whose children had donated organs expressed different elements of grief to what hospice staff were used to dealing with. In addition, whilst hospice staff are used to caring for a child's body after death in a cool bedroom, the surgical wounds that resulted from organ retrieval increased the anxiety of staff. Some staff were also anxious that broadening the criteria to care for children who did not have a known LLC after death would result in big increases in the use of the cool rooms, changing the 'feel' of the hospice, impacting on the care of existing families who use the hospice for short breaks, symptom control and end of life care.

'it sounds crazy to say this now, but I remember talking to colleagues about how nervous we were about opening up the [cool room] to more children - how would we cope with caring for more families who are going through something very different than what we're used to. 
Organ donation and children's hospice cool rooms.

Also, what would it mean for families already here - they were used to the hospice being a happy place, not a mortuary' (HN2)

Hospice staff talked about quickly realising that the bereavement care needs of families of children who had donated organs was not significantly different to those of families whose children had a progressive LLC. Hospice staff acknowledge that the concept of broadening the cool room acceptance criteria was worse than the reality. Comparisons were drawn with other changes implemented over the last few years, including changes to the hospice's referral criteria, discussing how increased exposure to any element of care led to competence and confidence within the nursing team.

\section{Influencers and enablers}

Actions and practices that influenced and maintained the changes to practice were discussed, acknowledging the education initiatives that had taken place between the hospice, SNOD team and regional PICU. The opportunity to understand the journey of families from hospital admission to bereavement follow up was highlighted by staff from both organisations as beneficial, as it allowed them to provide information to families, and improved support offered due to increased awareness of what families has experienced from other, allied services:

'The training on organ donation was great - I had no idea that is what families went through. I often think about that when I'm with a family of a child - to know what happened in hospital helps me to support them.' (HN1)

The whole SNOD Team, comprising 21 nurses, have visited the hospice, allowing them to see the building and the care offered, with particular emphasis on the cool rooms, and bereavement support offered. The 'mystery' of children's hospices was discussed by SNODs and acknowledged by hospice staff, around how professionals are often unaware of what care is offered in a children's hospice. 
Organ donation and children's hospice cool rooms.

SNODs acknowledged that as a predominantly adult-trained team, paediatric donors 'frightened' them, and required an enhanced 'focus on families' in a different way to adult donors, and how this can be achieved effectively by a children's hospice:

'The hospice is amazing, I had no idea it would be like this. It's not what you imagine at all, is it? I mean, I would never have thought to refer to [the hospice] if I hadn't had that training, and now I can't think of anywhere better.' (SNOD3)

The interagency, multiprofessional training, and the opportunity to visit the hospice were highlighted by both groups of nurses for increasing the awareness of the roles of colleagues across the organisations. SNODs discussed how this has resulted in them feeling confident to ring the hospice with potential referrals, to seek advice for children who live outside of the hospice catchment area, or to discuss bereavement support for families who are not able to, or choose not to use the cool rooms.

'We know we can pick the phone up and ring you now - we don't need to know if a certain person is in, but that any of the team will help - you're all lovely and know what you're talking about!' (SNOD1)

\section{Sustainability}

Sustainability was discussed from the perspective of both SNODs and hospice staff. SNODs considered the recent changes to centralising some of the administration around contacting donor families, a role previously undertaken by the nurse who supported the family before and during donation. They also highlighted how the increased numbers of donors meant that SNODs have less time to spend with families.

'Unfortunately, you end up getting so busy with other families, and obviously for them, they're going through it, they're in that bubble, and then for us, we're having to deal with so 
Organ donation and children's hospice cool rooms.

many other families in their position, but [to an individual family], they are the most important people in this.' (SNOD1)

The sustainability of the new, broader scope was questioned by hospice staff, in terms of burn out, due to fears around being exposed to 'traumatic' deaths and grief, and the number of families who may use the service.

'there were a group of us who were worried that we were opening the flood gates - we'd never done anything like this before, and once you've offered it to one, you have to offer it to all'. (HN3)

Nurses from both organisations recognised the benefit of the new service to families, and were really pleased to be able to respond to contemporary need.

'This is what we've always said we do - care for families whose child has died. The circumstances may be different, but essentially, we're doing what we've always done - caring for bereaved families.' (HN1)

'It's one of those things you think 'I can't believe we haven't always done it like this', but it's not until you see it that you can understand it.' (SNOD1)

\section{Discussion}

This study explored the perspectives of organ donation nurses and children's hospice nurses who had been involved in the care of the bodies of children, and their families, after donating organs. Five themes emerged from a semi-structured focus group: barriers, bereavement, impact, influencers and enablers, and sustainability. Changes to the SNOD role led the team to recognise the barriers that prevented them from providing the level of bereavement support they felt paediatric donor families needed, and to seek alternative support for the families of children. The 
Organ donation and children's hospice cool rooms.

bereavement support offered by both the SNOD team and the hospice were recognised and valued by the other organisation, however the long-term, responsive and family centred approach to bereavement support was regarded as a strength of the hospice model. Nurses perceived the impact of the service change was threefold: families benefited from the responsive, personalised and family-focused bereavement support offered by the hospice. This led to SNODs feeling that families were being supported effectively, bringing a sense of relief to the SNODs, reducing moral distress. Initially, hospice staff found the addition of caring for children with variant conditions difficult, but developed confidence as the number of referrals increased. An awareness of the roles of all professionals and teams involved in the 'organ donation journey' was acknowledged as an enabler, achieved through joint training and tailored hospice visits; these were noted as being particularly helpful to SNODs whose professional background was predominantly adult critical care nursing. Sustainability was important to nurses from both organisations; SNODs recognised that the previous approach was unsustainable and that change was needed to reflect family needs. Hospice nurses were keen to offer consistent, equitable support to the families of children who had donated organs, without compromising the care of families who utilised the conventional care services offered by the hospice. We are not aware of any other piece of research that has explored this phenomenon.

Decisions around a child's end of life care are likely to be the most difficult choices parents will ever make (Bellali \& Papadatou 2006), making the difficult and selfless decision to donate organs incredibly hard. However, the opportunity to donate their child's organs has been shown to have a positive impact on the bereavement journey of parents whose child has died (Vitali and Burns, 2015). The participants in this study recognised the bereavement needs of families, in addition to the selflessness they showed by donating their child's organs in order to help others, and were committed to providing excellent care. Ahmadian, Rahimi, and Khaleghi (2017) found that families face a wide range of challenges post-donation, which together with the grief in response to their 
Organ donation and children's hospice cool rooms.

child's death can complicate bereavement. This demonstrates a clear need for ongoing bereavement support for this group of families, which is not currently being met by existing organ donation services.

Organ donation is critically connected with sudden death (Walker \& Sque 2016); the ability to provide appropriate and timely support to families has shown to have a positive effect on bereavement outcomes (Sque et al. 2018). Families' experience of dissonance is reported by Mills and Koulouglioti (2016), owing to the lack of information that is often available. The multi-layered roles of nursing patient's families is highlighted, particularly in relation to providing information around the patient's condition, treatment options and possible outcomes, as well as providing emotional support (Mills and Koulouglioti, 2016). The family-centred nature of both hospice and organ donation nursing was clear throughout the focus group, discussed by all participants. There was a definite will to provide the best possible outcomes for families of deceased children expressed by nurses from both organisations, juxtaposed against their current role, the needs of an individual family, and other families cared for by their organisations. The balancing of these components appeared to produce a degree of moral distress in participants, associated with sadness, anger, anxiety, helplessness, feelings of failure, shame and anguish (Thorne, Konikoff, Brown, and Albersheim, 2018). Current evidence suggests that beyond the effects on individuals, high levels of moral distress can compromise care quality, including avoidance behaviours toward patients, absenteeism, poor staff retention, low staff morale and poor staff cohesion (Thorne et al. 2018). A number of barriers were discussed by the SNODs, many of these relating to organisational changes, many of which are reported throughout the National Health Service, such as reduced number of nurses, and nursing levels that do not match the population of service demand (Murray 2017). The findings of this study suggest that moral distress of staff is reduced, as they feel that families are receiving the care they deserve. 
Organ donation and children's hospice cool rooms.

Changes in family composition was noted by both SNODs and hospice nurses, particularly the notion of blended families and the inclusion of wider family members in key decision making relating to care, who are being seen with increased frequency by the organ donation team. The perpetual evolution of family centred care, evolving to reflect the context of family makes defining it challenging (Smith and Coleman, 2010), however it remains a fundamental principle on which children's nursing is based in the UK (Dennis et al. 2017). The flexibility shown by children's hospices in their approach to all aspects of palliative care provision mean that they are well-equipped to meet the needs of contemporary families, including blended and separated families, and wider family members, including siblings and grandparents (Kirk \& Pritchard 2012).

\section{Limitations of the study}

Despite providing valuable insight into how registered nurses perceive the impact of caring for the families of children who have donated organs, the limitations of this study should be acknowledged. The study was confided to a single partnership between two organisations, thus caution should be exercised when applying the findings to other settings, although parallels have been drawn from the wider literature, demonstrating broader application of the findings.

As with any focus group, the possibility that the opinions of more forceful or articulate participants dominated the group or findings is a concern. Although this was considered when planning the study, there remains a chance that participant's contributions were shaped by what they anticipated would be favoured by fellow participants or the interviewers.

\section{Implications for practice and policy}

We believe this to be the first explorative study of its kind internationally, and that there are a number of implications for palliative care services, particularly those offering children's hospice care. 
Organ donation and children's hospice cool rooms.

\section{Clinical implications}

Bereaved families were perceived to benefit from the change in practice that enabled their child's body to be cared for at the hospice following organ donation, and access to the enhanced hospicebased bereavement support that followed. The interagency working described in this paper improved the support families are offered following the death of their child, recognising the contributions and limitations of each organisation transparently.

\section{Policy implications}

This paper highlights that the bereavement outcomes of families are affected by the level and quality of bereavement care they are offered post donation, as such the implications for policy are:

- A divergence away from traditional hospice acceptance criteria, to reflect the needs of local families and service allowed care to be provided to families who would otherwise not be enabled to access the bereavement support they need. This was achieved through interagency collaboration.

- Services that meet the needs of contemporary families are vital if the care is to be effective and beneficial.

- Interagency pathways for bereavement support, which allow a multiagency approach, recognising the contributions and limitations of care

- The importance of post-donation bereavement support is required

- The admission policies of children's hospice will need to be broadened to include children who were previously unknown to them following organ donation.

\section{Conclusions}

Although organ donation nurses seek to provide meaningful post-donation bereavement support, organisational constraints, increases in the number of referrals for donation and the large geographical regions covered by SNODs mean that they are unable to do so, resulting in families not 
Organ donation and children's hospice cool rooms.

receiving the care that SNODs want to be able to provide, leading to moral distress. The bereavement needs of families, which are shown to be complicated post-donation, and affected by the levels of support they receive are largely left unmet. SNODs reported substantial emotional and moral distress owing to their inability to meet the expressed needs of families, due to organisational constraints. This interagency initiative is an example of how, by working across organisational boundaries, relevant care can be provided to families, supporting them post-donation, reflecting contemporary need and providing an increased sense of role satisfaction in nurses who support a family at any stage in their organ donation journey. 